\section{S17.2 HPV INFECTION, GENITAL INFLAMMATION, AND HIV RISK IN THE CAPRISA 004 TRIAL}

${ }^{1,4}$ Quarraisha Abdool Karim, 'Lenine J Liebenberg*, 'Kerry Leask, ${ }^{1}$ Natasha Samsunder, ${ }^{1}$ Ayesha Kharsany, ${ }^{1}$ Anneke Grobler, 'Lyle McKinnon, 1,3,5Jo-Ann Passmore, ${ }^{1,4}$ Salim Abdool Karim. ${ }^{1}$ Centre for the AIDS Programme of Research in South Africa, Durban, South Africa; ${ }^{2}$ National Institute for Communicable Diseases; ${ }^{3}$ Infectious Disease and Molecular Medicine, University of Cape Town, Observatory, Cape Town, South Africa; ${ }^{4}$ Department of Epidemiology, Mailman School of Public Health, Columbia University, NY, USA; ${ }^{5}$ National Health Laboratory Services, Cape Town, South Africa

\section{$10.1136 /$ sextrans-2015-052270.76}

There is a growing body of data demonstrating an increased risk for HIV acquisition in the presence of HPV infection, but the mechanism of this relationship is unclear. This study investigated the impact of HPV infection on both genital inflammation and HIV risk in the CAPRISA 004 1\% TFV gel trial.

Baseline cervicovaginal lavage specimens collected from 737 HIV-uninfected women were analysed to determine the prevalence of HPV infection. Clinical, reproductive, demographic and behavioral data were captured. The presence of DNA from 37 HPV genotypes was assessed using Linear Array, and the concentrations of 48 relevant cytokines were quantified by multiplexed ELISA assays. The presence of HIV was measured monthly using two rapid tests and confirmed by western blot and PCR.

Of the 737 eligible participants, 74\% had prevalent HPVinfection (95\% CI: 71-77\%). Participants with prevalent HPV infection were 2.8 times more likely to acquire HIV infection compared to those with no HPV infection (95\% CI: $1.3-5.9$; p $=0.007)$. HIV risk was independent of the oncogenicity of HPV strains at baseline [(HPV oncogenic strains HR $2.5(95 \%$ CI 1.0-6.2) vs non-oncogenic strains HR 2.1 (95\% CI 0.9-5.1)], and was also increased in the presence of multiple concurrent infections (HR 3.1; 95\% CI 1.4-6.8).

No cytokine signatures were associated with prevalent HPV infection. The use of tenofovir gel did not prevent HPV infection.

These data confirm a relationship between HPV infection and increased risk for HIV acquisition, and underscores the need to define the underlying biological mechanisms to inform targeted interventions in settings that bear a high burden of both infections.

\section{S17.3 NOVEL THERAPIES FOR HPV-RELATED ANAL DISEASE}

Henry de Vries*. Public Health Service Amsterdam, University of Amsterdam, Amsterdam and National Institute for Public Health and the Environment, Bilthoven, The Netherlands

\subsection{6/sextrans-2015-052270.77}

Anal cancer is an increasing problem among patients with HIV, especially among HIV-positive men who have sex with men (MSM) with incidence rates 100 per 100,000 person-years. This is much higher than the incidence of cervical cancer in HIV-negative women before standard cytological screening was introduced. Therefore, routine screening for anal premalignant lesions is subject of discussion. As with cervical cancer, anal cancer is preceded by a precursor called anal intraepithelial neoplasia (AIN).

In this presentation I will discuss the standard therapeutic options practiced to treat AIN lesions with the goal to prevent progression towards invasive carcinoma. Electrocauterisation is the most used option to date for AIN lesions. Other ablative measures are infrared coagulation, highly concentrated trichloroacetic acid, and liquid nitrogen. Whereas ablative therapy has to be performed in an outpatient clinical setting by trained health workers, patient administered home based treatment options have been studied also, like imiquimod, and 5 -fluorouracil cream. Until now most treatment modalities have been studied in open label and/or non-randomised trials, and very little comparative studies have been performed. Therefore, scarce evidence-based data on the treatment of AIN is available. It is likely that intra-anal versus perianal AIN disease requires different treatment approaches.

So far, most AIN treatment studies show high recurrency rates, irrespective of the modality used. Novel treatment options are therefore looked after. Vaccination studies, using both preventive and therapeutic vaccines are ongoing. Preventive vaccines, targeting the causative HPV virus have been tried to prevent recurrent disease in successfully treated AIN patients. In established disease, HPV preventive vaccines are not believed to be effective since HPV E6 and E7 viral genes by then are incorporated in the host genome. Therefore, therapeutic AIN vaccines will need to target the transfected cell directly. Studies with E6 and/or E7 DNA targeting vaccines are ongoing.

\section{S17.4 USING DATA ON PATHOGENESIS AND EPIDEMIOLOGY TO INFORM ANAL CANCER SCREENING STRATEGIES: DATA FROM THE STUDY OF PREVENTION OF ANAL CANCER (SPANC)}

Mary Poynten*, On behalf of the SPANC study team. The SPANC study team includes Andrew Grulich, Mary Poynten, Jeff Jin, David Templeton, Garrett Prestage, Dorothy Machalek, (Kirby Institute, UNSW, Sydney, Australia); Andrew Carr, Winnie Tong, (St Vincent's Hospital, Sydney); Chistopher Fairley (Melbourne Sexual Health Centre, Melbourne); Richard Hillman, Kirsten Howard, Kirsten McCaffery (Sydney University); Annabelle Farnsworth, Jennifer Roberts (Douglas Hanly Moir Pathology, Sydney); Suzanne Garland, Sepehr Tabrizi, Alyssa Cornall (Royal Women's Hospital, Melbourne); Geoff Honnor, Kathy Triffit (Community representatives)

\section{$10.1136 /$ sextrans-2015-052270.78}

Background HPV vaccination of school-aged boys will prevent anal cancer in future generations. Vaccination of gay men up to age 26 is recommended in several jurisdictions, but vaccination is generally not recommended at older ages because of a concern of possible lack of efficacy due to past or current HPV infection. Anal cancer screening, based on the model of cervical cancer screening, has also been proposed as a means to reduce morbidity.

Methods The Study of the Prevention of Anal Cancer (SPANC) is a three-year prospective study of the natural history of anal HPV infection and cancer precursors in HIV-negative and -positive gay men aged $\geq 35$ years. At each visit all men receive an anal swab for cytology and HPV genotyping, followed by high resolution anoscopy-directed biopsy for histological assessment.

Results At the end of June 2015, 595 men had been enrolled. Median age was 49 and $35.3 \%$ were HIV-positive. Men of all ages enrolled in SPANC were likely to report multiple sexual partners in the past 6 months (overall $73.4 \%$ of $35-44$ year olds decreasing to $62.1 \%$ of $65+$ year olds, $\mathrm{p}$ trend $=0.03$ ). The prevalence of HPV16, the genotype responsible for $>90 \%$ of anal cancer, was $29.4 \%$ in $35-44,30.8 \%$ in $45-54,34.2 \%$ in $55-64$ and $19.0 \%$ in $65+$ year olds ( $\mathrm{p}$ trend $=0.54$ ), with no difference by HIV status. The incidence of HPV16 decreased with age from 5.6/100 person years (PY) in 35-44 year olds to 2.9/100PY in 55-64 year olds. There was no incident HPV16 in men aged $65+(\mathrm{p}$ trend $=0.059)$. At baseline, the prevalence of 
anal high grade intraepithelial neoplasia (HSIL) was higher in HIV-positive than in the HIV-negative participants $(46.9 \%$ vs $32.3 \%, \mathrm{p}<0.001)$. Among those with HSIL at baseline, the clearance rate was similar between HIV-positive and -negative participants (38.4 vs 38.0 per 100PY, $\mathrm{p}=0.636$ ).

Conclusions Gay men in SPANC reported multiple sexual partners across the adult age-range, and incident HPV16 continued to be detected in men up to their seventh decade of life. This suggests that HPV vaccination of adult gay men may prevent infection and have a role in cancer prevention. Anal HSIL is highly prevalent, particularly among HIV-positive men, but there are high rates of clearance without treatment. These data suggest that a screening test which can distinguish persistent from transient HSIL is required. The role of HPV biomarkers in identifying those HSIL lesions most likely to persist should be investigated.

\section{Oral Presentations}

\section{1 - Spread of antimicrobial-resistant gonorrhoea}

\subsection{EVOLUTION AND SPREAD OF ANTIBIOTIC-RESISTANT GONORRHOEA}

${ }^{1,2} \mathrm{SM}$ Fingerhuth*, ${ }^{2} \mathrm{~S}$ Bonhoeffer, ${ }^{1} \mathrm{~N}$ Low, ${ }^{1} \mathrm{CL}$ Althaus. ${ }^{1}$ Institute of Social and Preventive Medicine (ISPM), University of Bern, Bern, Switzerland; ' Institute of Integrative Biology, ETH Zurich, Zurich, Switzerland

\subsection{6/sextrans-2015-052270.79}

Introduction Neisseria gonorrhoeae has developed resistance to all classes of antimicrobials that have been used to treat it and strains that are resistant to multiple classes of antimicrobials have evolved. Only one antimicrobial (ceftriaxone) can now be used for empirical treatment in many countries. Hence, it is important to understand the dynamics and drivers of resistance evolution.

Methods First, we estimated rates of resistance evolution from antimicrobial resistance surveillance data from the USA and from England and Wales for heterosexual men (HetM) and men who have sex with men (MSM). Second, we developed dynamic transmission models to reconstruct the observed dynamics of $N$. gonorrhoeae transmission and resistance evolution in both HetM and MSM.

Results We found that resistance to ciprofloxacin and cefixime initially spreads exponentially at rates between 0.2 and 2.4 per year. These rates suggest that the proportion of resistant strains doubles every 3 to 35 months. We found lower rates of spread in HetM (0.2 to 0.8) compared with MSM (0.9 to 2.4). The models show that the treatment rate is the driving force for the spread of resistance.

Conclusion There is a trade-off in optimising the treatment rate to provide individual patient care to all those who are infected and to keep the spread of resistance as low as possible. These findings have implications for developing antimicrobial treatment strategies and point-of-care tests to detect resistance.

Disclosure of interest statement This study received support from the RaDAR-Go (Rapid Diagnosis of Antimicrobial Resistance in Gonorrhoea) project, funded by SwissTransMed and from the Swiss National Science Foundation. No pharmaceutical grants were received for the conduct of this study.
001.2 AZITHROMYCIN-RESISTANT NEISSERIA GONORRHOEAE IN MEN WHO HAVE SEX WITH MEN (MSM) IN SEATTLE, WASHINGTON: 2014-2015

1,2 LA Barbee*, 1,300 Soge, ${ }^{1,2} \mathrm{JC}$ Dombrowski, ${ }^{1,2}$ DA Katz, ${ }^{1,3} \mathrm{KK}$ Holmes, ${ }^{1,2} \mathrm{MR}$ Golden. ${ }^{1}$ University of Washington; ${ }^{2}$ Public Health - Seattle \& King County; ${ }^{3}$ Neisseria Reference Laboratory

10.1136/sextrans-2015-052270.80

Background The emergence of azithromycin-resistant Neisseria gonorrhoeae threatens recommended first-line treatment regimens.

Methods We investigated cases of azithromycin-resistant gonorrhoea detected at Public Health - Seattle and King County STD Clinic between January 2014 and February 2015. In 20122013, zero of 237 MSM urethral isolates were azithromycinresistant. The US Centres for Disease Control and Prevention (CDC) and Clinical and Laboratory Standards Institute (CLSI) do not define an azithromycin-resistance breakpoint. CDC uses $\geq 2 \mathrm{mcg} / \mathrm{mL}$ as an "Alert Value" minimal inhibitory concentration (MIC) (here called resistant). We used agar dilution to determine MICs.

Results Of 179 urethral, 83 pharyngeal and 87 rectal isolates from MSM, 11 (6.1\%), 6 (7.2\%) and 5 (5.7\%) had azithromycin MIC $\geq 2 \mathrm{mcg} / \mathrm{mL}$, respectively. We identified no cases of azithromycin-resistance among 56 heterosexuals. Overall, 19 (6.9\%) of 276 MSM with culture-positive gonorrhoea had an azithromycin-resistant isolate. The median azithromycin MIC was 4.0 $\mathrm{mcg} / \mathrm{mL}$ (range 2 to $>256 \mathrm{mcg} / \mathrm{mL}$ ). Eight patients' isolates also demonstrated tetracycline resistance (MIC $\geq 2 \mathrm{mcg} / \mathrm{mL}$ ); one was ciprofloxacin-resistant (MIC $16.0 \mathrm{mcg} / \mathrm{mL}$ ). None exhibited reduced susceptibility to cefixime or ceftriaxone. Clinicians treated thirteen $(68 \%)$ cases with ceftriaxone and azithromycin, one (5.3\%) with ceftriaxone and doxycycline, two (10.6\%) with study drug, and three (15.8\%) with $2 \mathrm{~g}$ of azithromycin. Two of three men treated with azithromycin-monotherapy had a test of cure (TOC) and both had persistent infection; their isolates demonstrated azithromycin MICs of $32 \mathrm{mcg} / \mathrm{mL}$ and $4.0 \mathrm{mcg} / \mathrm{mL}$. One rectal treatment-failure was cured with $360 \mathrm{mg}$ of gentamicin intramuscularly; the other (urethra/pharynx positive), was treated with ceftriaxone and doxycycline. He did not undergo repeat TOC. The individual with high-level azithromycin-resistance (MIC $>256 \mathrm{mcg} / \mathrm{mL}$ ) was an international traveller and not locatable for TOC.

Conclusions Clinically important azithromycin-resistant Neisseria gonorrhoeae now are found in $>5 \%$ of MSM with gonorrhoea in Seattle. These findings support new CDC recommendations to avoid treating gonorrhoea with azithromycin alone.

Disclosure of interest statement This work was funded by the US National Institutes of Health and CDC. No pharmaceutical grants were received in the development of this study.

\subsection{HIGH-LEVEL AZITHROMYCIN RESISTANCE IN NEISSERIA GONORRHOEAE CLINICAL ISOLATES IN NANJING, CHINA, 2013-2014}

${ }^{1}$ Xiaohong Su, ${ }^{1}$ Wenjing Le, ${ }^{1}$ Yurong Liu, ${ }^{1}$ Chuan Wan, ${ }^{1}$ Sai Li, 'Baoxi Wang, ${ }^{2}$ Peter A Rice. ${ }^{1}$ National Center for STD and Leprosy Control, Institute of Dermatology, Chinese Academy of Medical Sciences \& Peking Union Medical College, Nanjing 210042, China; ${ }^{2}$ Division of Infectious Diseases and Immunology, University of Massachusetts Medical School, Worcester, MA 01602, USA

10.1136/sextrans-2015-052270.81 